LPTENS-06/10

\title{
Asymptotic Bethe Ansatz from String Sigma Model on $S^{3} \times R$
}

\author{
Nikolay Gromov ${ }^{a, b}$, Vladimir Kazakov ${ }^{a, *}$ \\ a Laboratoire de Physique Théorique \\ de l'Ecole Normale Supérieure et l'Université Paris-VI, \\ Paris, 75231, France ${ }^{\dagger}$ \\ ${ }^{b}$ St.Petersburg INP, \\ Gatchina, 188 300, St.Petersburg, Russia
}

\begin{abstract}
We derive the asymptotic Bethe ansatz (AFS equations 1]) for the string on $S^{3} \times R$ sector of $A d S_{5} \times S^{5}$ from the integrable nonhomogeneous dynamical spin chain for the string sigma model proposed in [2]. It is clear from the derivation that AFS equations can be viewed only as an effective model describing a certain regime of a more fundamental inhomogeneous spin chain.
\end{abstract}

\footnotetext{
* Membre de l'Institut Universitaire de France

†Unité mixte de Recherche du Centre National de la Recherche Scientifique et de l'Ecole Normale Supérieure associée à l'Université de Paris-Sud et l'Université Paris-VI gromov@thd.pnpi.spb.ru, nik_gromov@mail.ru kazakov@physique.ens.fr
} 


\section{Introduction}

Recently, it was proposed 22 to describe the compact, $R \times S^{5}$ sector of the Green-Schwarz-Metsaev-Tseytlin (GSMT) superstring on $A d S_{5} \times S^{5}$ background by the inhomogeneous, dynamical spin chain (we will abbreviate it to IDSC) built out of the physical particles of the quantum $S O(6)$ sigma model. The particles obey Zamolodchikovs factorizable S-matrix [3] and are put on the space circle representing the worldsheet direction of the closed string in the conformal gauge. Their isotopic $S O(6)$ vector degrees of freedom realize the target space projection to $S^{5}$. The proposal of [2] was inspired by a similar construction of 4], who considered a different, supersymmetric conformal $O S P(2 m+2 \mid 2 m)$ sigma-model.

This sigma model is asymptotically free and cannot pretend to the precise quantum description of the GSMT superstring. However, as was shown in [2], the Bethe ansatz equations of the model perfectly reproduce in the classical limit the finite gap solutions of [5] [6] in terms of their algebraic curve ${ }^{1}$.

A natural question can be posed about the proposal of [2]: does it capture some essential features of the quantum superstring theory? For the moment we dispose a very limited information about the quantum behavior of GSMT superstring. The only robust calculations are made in the so called BMN limit [7] where the quantum one loop $1 / \sqrt{\lambda}^{2}$ corrections were calculated [8], as well as the same corrections around the classical solutions for the simplest string motions: for the rotating circular and folded string [9, 10, 11.

In an interesting attempt to quantize the string on $R \times S^{3}$, the authors of [1 conjectured a discretization of the classical finite gap equations of [5] which in addition has the right BMN and the large gauge coupling limits. It was later pointed out [12, 13] that the resulting equation for Bethe roots eq. (20) (the so called AFS equation) can capture only a part of the truth, having chances to describe only large $\lambda$ and large angular momentum $L$ of the string on $S^{3}$ ).

Nevertheless, the AFS equations appeared to be a useful empirical guideline for the search of quantum formalism based on integrability. First, they appear to be the Bethe ansatz equations of a certain nonlocal spin chain [30. Second, they were generalized in a natural way to the full superstring theory in [14, following nice observations of [15]. This general model appears to be different from the asymptotic SYM Bethe ansatz equation eq. (19), the so called BDS equation, proposed in [16], the so called BDS equation, only by a universal scalar factor $\sigma^{2}$ eq. (21). The BDS is better justified and to great extent even deduced from the superalgebra of SYM theory [18, 19]. The BDS equation is claimed to be constrained by the crossing relations [20. It reproduces correctly at least three loops of anomalous dimensions of SYM theory [15]. Moreover, the quantum corrections of [9, 10, 11] were reproduced on the basis of AFS equations with some modifications in [13, 21, 22, 23. All this means that the empirical AFS equations contain some grain of truth about the structure of the quantum superstring.

The main problem with AFS equations is that they are not deduced from any general enough principle as an approximation with respect to a parameter, but rather proposed as an empirical guess.

In this paper, we will show that the AFS equation eq. (43) follows for large $\lambda$ and $L$ from the integrable quantum inhomogeneous dynamical spin chain (IDSC) of [2] based on the sigma model on $S^{3}$. The IDSC plays similar role for the AFS equation as the Hubbard model [17] for the BDS equation of [16. The derivation is quite straightforward and it demonstrates the qualitative nature of the AFS equations, explicitly containing large parameters $L$ and $\lambda$. The IDSC model seems to be more simple and fundamental since it is a selfconsistent integrable quantum system. It may incorporate the known data for both perturbative small $\lambda$, SYM theory and the large $\lambda$ quasiclassical string results since it has two a priory adjustable functions, dispersion of the particles and the scalar S-matrix factor, which can differ from the Zamolodchikovs form and describe a different quantum physics.

The paper is organized as follows. In section 2 we remind the classical formulation of sigma model on $S^{3}$, its IDSC quantization, as well as the AFS asymptotic string Bethe ansatz and the asymptotic BDS SYM equations. In section 3 we derive the AFS Bethe equations from the IDSC model and discuss a

\footnotetext{
${ }^{1}$ In 4 it was done for the $\operatorname{OSP}(2 m+2 \mid 2 m)$ sigma model, but the details of the classical limit are there quite different from ours.

${ }^{2} \lambda$ is the 't Hooft coupling constant of the dual $N=4$ SYM theory; $\hbar=1 / \sqrt{\lambda}$ plays the role of the Planck constant of the worldsheet sigma model.
} 
possible relation to BDS equations. The section 4 is devoted to discussion and conclusions. The details of the derivation of AFS formula are given in the Appendices A,B. The Appendices C, D are devoted to the generalization to the full scalar sector. ${ }^{3}$ In Appendix E we give simplified derivation of the classical limit of [2].

\section{$2 \quad$ String on $S^{3} \times R$ space as inhomogeneous spin chain}

\subsection{The sigma-model on $S^{3} \times R$}

The action of the $S^{3} \times R_{t}$ reduction of Green-Schwarz-Metsaev-Tseytlin superstring on $A d S_{5} \times S^{5}$ background [26. 27] in the conformal gauge in terms of homogeneous target space coordinates $X_{i}(\tau, \sigma), i=1, \ldots, 4$ and a scalar $Y(\tau, \sigma)$ representing the AdS time looks as follows ${ }^{4}$ :

$$
S=\frac{\sqrt{\lambda}}{4 \pi} \int_{0}^{2 \pi} d \sigma \int d \tau\left[\left(\partial_{a} X_{i}\right)^{2}-\left(\partial_{a} Y\right)^{2}\right], \quad X_{i} X_{i}=1
$$

The Virasoro conditions in the gauge

$$
Y=\kappa \tau
$$

read

$$
\left(\partial_{ \pm} X_{i}\right)^{2}=\left(\partial_{ \pm} Y\right)^{2}=\kappa^{2}, \partial_{ \pm}=\partial_{\tau} \pm \partial_{\sigma}
$$

$\Delta=\sqrt{\lambda} \kappa$ is identified with the dimension of the corresponding SYM operator according to the AdS/CFT correspondence.

After the gauge is imposed, the model looks like the $O(4)$ sigma model. The action of the theory can be represented in terms of the $S U(2)$ group valued field $\hat{g}=X_{1}+i \tau_{3} X_{2}+i \tau_{2} X_{3}+i \tau_{1} X_{4}\left(\tau_{i}\right.$ are the Pauli matrices). Then the action eq. (11) takes the form of the $S U(2)$ principal chiral field

$$
S=\frac{\sqrt{\lambda}}{4 \pi} \int_{0}^{2 \pi} d \sigma \int d \tau\left(\partial_{a} X_{i}\right)^{2}=-\frac{\sqrt{\lambda}}{8 \pi} \int d^{2} \sigma \operatorname{tr}\left(j_{a}^{L, R} j_{a}^{L, R}\right)
$$

where

$$
j_{ \pm}^{R}=\hat{g}^{-1} \partial_{ \pm} \hat{g}, \quad j_{ \pm}^{L}=\partial_{ \pm} \hat{g} \hat{g}^{-1},
$$

are the currents of the global symmetry of left and right multiplication by $S U(2)$ group element. The corresponding Noether charges are

$$
Q_{L}=\frac{\sqrt{\lambda}}{4 \pi} \int d \sigma \operatorname{tr}\left(i \partial_{0} \hat{g} \hat{g}^{\dagger} \tau^{3}\right), \quad Q_{R}=\frac{\sqrt{\lambda}}{4 \pi} \int d \sigma \operatorname{tr}\left(i \hat{g}^{\dagger} \partial_{0} \hat{g} \tau^{3}\right)
$$

From the action we read off the energy and momentum as

$$
E \pm P=-\frac{\sqrt{\lambda}}{8 \pi} \int \operatorname{tr}\left[j_{0} \pm j_{1}\right]^{2} d \sigma=\frac{\sqrt{\lambda}}{2} \kappa^{2}
$$

and in particular one has the level matching condition

$$
P=0
$$

\footnotetext{
${ }^{3}$ The result of this generalization is different from the asymptotic Bethe equations of 14, probably because it completely ignores the interactions with fermions and the noncompact sector; however, the formulas might be potentially useful for the search of the IDSC formulation of the full GSMT superstgring.

${ }^{4}$ The coupling constant in front of the action is identified by the AdS/CFT correspondence with the 't Hooft coupling $\lambda=g^{2} N_{c}$ of the $\mathcal{N}=4$ supersymmetric Yang-Mills (SYM) theory [10]
} 


\subsection{Inhomogeneous dynamical spin chain (IDSC)}

Of course, the sigma-model eq. (11) can be viewed as a consistent truncation of the full GSMT superstring to the $S^{3} \times R$ sector only classically. In the full quantum sigma-model one cannot turn off the interactions with fermions, and through them, with all the rest of the bosonic coordinates of the string. On the other hand, in the dual $N=4 \mathrm{SYM}$ theory the $S U(2)$ sector seems to be closed at least perturbatively. So one may hope that one could capture the essential features of the quantum string sigma model by considering quantization of the pure $O(4)$ sigma model eq.(11), or of the equivalent $S U(2)_{L} \times S U(2)_{R}$ principal chiral field, eq. (4).

The asymptotically free sigma-model eq.(1) is integrable and obeys the factorizable S-matrix for its physical particles [3] (see 2] for the details). It was proposed in 2], following the analysis of a similar supersymmetric sigma-model in 4, to describe the quantum string as a collection of $L$ physical particles with rapidities $\theta_{k}, \quad k=1, \cdots, L$, on a circle of the length $2 \pi$ representing the periodic world sheet $\sigma$ coordinate of the closed string.

The periodicity condition on the circle for the Bethe wave function of these particles, written in terms of a system of Bethe equations (see the details in [2]), looks as follows

$$
e^{-i \mu \sinh \pi \theta_{\alpha}}|\psi\rangle=\prod_{1}^{\alpha-1} \hat{S}\left(\theta_{\alpha}-\theta_{\beta}\right) \prod_{N}^{\alpha+1} \hat{S}\left(\theta_{\alpha}-\theta_{\beta}\right)|\psi\rangle .
$$

The r.h.s. of these equations has a form of a spin chain transfer-matrix acting on an eigenvector $|\psi\rangle$ with eigenvalue $e^{-i \mu \sinh \pi \theta_{\alpha}}$. Since the matrices $\hat{S}$ describing the quantum spins at individual sites of such a spin chain depend on $\theta$ 's, which by themselves should be determined from this equation, we call this system inhomogeneous dynamical spin chain, or IDSC for short ${ }^{5}$.

The eigenvalue problem eq. (9) can be solved by the standard algebraic Bethe ansatz techniques and gives the following system of nested Bethe ansatz equations

$$
\begin{aligned}
e^{-i p\left(\theta_{\alpha}\right)} & =\prod_{\beta \neq \alpha} S_{0}^{2}\left(\theta_{\alpha}-\theta_{\beta}\right) \prod_{j} \frac{\theta_{\alpha}-u_{j}+i / 2}{\theta_{\alpha}-u_{j}-i / 2} \prod_{k} \frac{\theta_{\alpha}-v_{k}+i / 2}{\theta_{\alpha}-v_{k}-i / 2}, \\
1 & =\prod_{\beta} \frac{u_{j}-\theta_{\beta}-i / 2}{u_{j}-\theta_{\beta}+i / 2} \prod_{i \neq j} \frac{u_{j}-u_{i}+i}{u_{j}-u_{i}-i} \\
1 & =\prod_{\beta} \frac{v_{k}-\theta_{\beta}-i / 2}{v_{k}-\theta_{\beta}+i / 2} \prod_{l \neq k} \frac{v_{k}-v_{l}+i}{v_{k}-v_{l}-i}
\end{aligned}
$$

where

$$
\alpha, \beta=1, \ldots, L, \quad i, j=1, \ldots, J_{u}, \quad k, l=1, \ldots, J_{v} .
$$

For the pure $S O(4)$ sigma model we should take the relativistic momentum dispersion for the physical particles

$$
p\left(\theta_{\alpha}\right)=\mu \sinh \pi \theta_{\alpha}
$$

and the Zamolodchikovs S-matrix scalar factor

$$
S_{0}(\theta)=i \frac{\Gamma\left(-\frac{\theta}{2 i}\right) \Gamma\left(\frac{1}{2}+\frac{\theta}{2 i}\right)}{\Gamma\left(\frac{\theta}{2 i}\right) \Gamma\left(\frac{1}{2}-\frac{\theta}{2 i}\right)}
$$

where

$$
\mu=e^{-\frac{\sqrt{\lambda}}{2}}=e^{-\frac{2 \pi g}{\sqrt{2}}}
$$

according to the asymptotic freedom.

\footnotetext{
${ }^{5}$ We hope to avoid the confusion with the dynamical spin chain with a changing length of 31]
} 
Energy and momentum are given by standard relativistic relations

$$
\begin{aligned}
& P=\frac{\mu}{2 \pi} \sum \sinh \left(\pi \theta_{\alpha}\right), \\
& E=\frac{\mu}{2 \pi} \sum \cosh \left(\pi \theta_{\alpha}\right) .
\end{aligned}
$$

In what follows we consider the limit of large $g$. Rapidities $\theta_{\alpha}$ can be considered as coordinates of particles in an external potential $\mu \cosh \pi \theta_{\alpha}$ with interaction

$$
i \log S_{0}^{2}(\theta) \simeq 1 / \theta+\mathcal{O}\left(1 / \theta^{3}\right) .
$$

When $g$ is large $\mu$ is exponentially small according to eq. (15) and the external potential becomes a square box potential with infinite walls at $\theta= \pm \sqrt{2} g$. Since the size of the box is large we can leave only the first, "Coulomb" term in interaction (18) between the particles. We will consider only the states with all $\theta$ 's having the same mode number $m$ (see eq.(29)). It was argued in 2 that such a selection of the states corresponds to the absence of longitudinal oscillations of the string, which is well seen at least in the classical limit characterized by a big number of large $\theta$ 's. Let us note that the $\theta$ excitations correspond roughly to the unphysical longitudinal motions of the string (which are excluded by the choice of the single mode number) and the magnon variables correspond to the transverse motions, which is well seen at least in the classical limit (see [2]).

Strictly speaking, we can trust the periodicity conditions eq. (9) only in the limit when the energies of individual particles is large $E_{\alpha}=\frac{\mu}{2 \pi} \cosh \left(\pi \theta_{\alpha}\right) \rightarrow \infty$, up to the terms $\sim e^{-E_{\alpha}}$. In the classical limit the total energy is large, but the energies of individual particles are in our case small for large $\lambda$. In that case the very notion of S-matrix looses its sense, since it is valid only in the infinite space, or at least when the size of the space is much bigger than the invers mass. Nevertheless the classical limit of [2] works well ${ }^{6}$. It might be that the physical interpretation in terms of the worldsheet particles is not adequate for the string and the right interpretation is in terms of the inhomogeneous dynamical quantum spin chain with the transfer matrix given by the r.h.s. of eq. (91), with the functions $p(\theta)$ and $S_{0}(\theta)$ yet to fix.

\subsection{AFS and BDS equations and notations}

The AFS equations for the energy spectrum of the $S^{3}$ subsector of the string on $A d S_{5} \times S^{5}$ [1] and BDS equations for the anomalous dimensions of $S U(2)$ subsector of $\mathcal{N}=4$ SYM theory [16] look very similar. For YM it reads

$$
\left(\frac{y_{k}^{+}}{y_{k}^{-}}\right)^{L}=\prod_{j \neq k}^{K} \frac{u_{k}-u_{j}+i}{u_{k}-u_{j}-i}
$$

for string one has to multiply r.h.s. by a "dressing" factor $\sigma^{2}$

$$
\left(\frac{y_{k}^{+}}{y_{k}^{-}}\right)^{L}=\prod_{j \neq k}^{K} \frac{u_{k}-u_{j}+i}{u_{k}-u_{j}-i} \sigma^{2}\left(u_{j}, u_{k}\right)
$$

where

$$
\sigma^{2}\left(u_{j}, u_{k}\right)=\left(\frac{1-1 /\left(y_{j}^{-} y_{k}^{+}\right)}{1-1 /\left(y_{j}^{+} y_{k}^{-}\right)}\right)^{-2}\left(\frac{\left(y_{j}^{-} y_{k}^{-}-1\right)}{\left(y_{j}^{-} y_{k}^{+}-1\right)} \frac{\left(y_{j}^{+} y_{k}^{+}-1\right)}{\left(y_{j}^{+} y_{k}^{-}-1\right)}\right)^{2 i\left(u_{j}-u_{k}\right)} .
$$

$y_{j}^{ \pm}$are defined by means of Zhukovsky transformation and its inverse

$$
Z(x) \equiv x+1 / x, \quad X(z) \equiv \frac{1}{2}\left(z+\sqrt{z^{2}-4}\right),
$$

\footnotetext{
${ }^{6}$ We thank Al. and A. Zamolodchikovs for the discussion on this point.
} 
where by definition we take the branch of the square root, so that $|X(z)|>1$ for $z \in \mathbb{C}$. Then

$$
y_{j}^{ \pm} \equiv X\left(\frac{u_{j} \pm i / 2}{M}\right)
$$

where $M$, introduced in [2], is

$$
M=\frac{g}{\sqrt{2}}=\frac{\sqrt{\lambda}}{4 \pi} .
$$

The systems of equations (1920) should be accompanied by the periodicity (level-matching) condition

$$
\sum_{j} \log \frac{y_{j}^{-}}{y_{j}^{+}}=2 \pi i m
$$

And finally,

$$
\Delta=L+2 i M \sum_{j=1}^{K}\left(\frac{1}{y_{j}^{+}}-\frac{1}{y_{j}^{-}}\right),
$$

giving us either the dimension of a SYM operator or the energy of a quantum string state.

\section{$3 \quad$ AFS equations from inhomogeneous spin chain}

If we put $J_{v}=0$ thus retaining only the excitations corresponding to the left charges in eq. (10)-(12) we obtain a reduced system of Bethe equations.

$$
\begin{aligned}
e^{-i p\left(\theta_{\alpha}\right)} & =\prod_{\beta \neq \alpha} S_{0}^{2}\left(\theta_{\alpha}-\theta_{\beta}\right) \prod_{j} \frac{\theta_{\alpha}-u_{j}+i / 2}{\theta_{\alpha}-u_{j}-i / 2}, \\
1 & =\prod_{\beta} \frac{u_{j}-\theta_{\beta}-i / 2}{u_{j}-\theta_{\beta}+i / 2} \prod_{i \neq j} \frac{u_{j}-u_{i}+i}{u_{j}-u_{i}-i},
\end{aligned}
$$

where

$$
\alpha, \beta=1, \ldots, L, \quad i, j=1, \ldots, J .
$$

Its classical limit reproduces the KMMZ finite gap equations which can be compared with the perturbatively closed $S U(2)$ sector of SYM.

Now we will show that in the continuous limit for $\theta$-distribution $L \rightarrow \infty$, and $g \sim L$, but for an arbitrary number $J_{u}$ of magnon variables $u_{k}^{7}$ the eq.(27)-(28) together with eqs. (16117) reproduce the AFS asymptotic Bethe ansatz from [1]. It will be clear from the derivation that the AFS equation plays only a role of an effective equation interpolating between BMN limit and classical limit. One could, for example, take into account $1 / \sqrt{\lambda}$ corrections stemming from the discreteness of the $\theta$ 's which are missing in AFS equation.

It will be important for us that in eq.(18) we take only the the first term, corresponding to the two dimensional Coulomb repulsion, and the $\theta$-variables will be confined in this limit, due to the dispersion eq.(13), in a square box with the vertical infinite walls at $\theta= \pm \sqrt{2} g$. It is clear from this that the characteristic $\theta$ 's should in principle scale as $\theta \sim \sqrt{\lambda} \rightarrow \infty$. The system eq. (27)-(28) becomes

$$
\begin{gathered}
f \frac{\rho_{\theta}(z) d z}{w-z}+2 \pi m=-i \sum_{j} \log \frac{w M-u_{j}+i / 2}{w M-u_{j}-i / 2}, \quad w \in(-2,2), \\
1=\prod_{\beta} \frac{u_{j}-\theta_{\beta}-i / 2}{u_{j}-\theta_{\beta}+i / 2} \prod_{k(\neq j)} \frac{u_{j}-u_{k}+i}{u_{j}-u_{k}-i} \\
\alpha, \beta=1, \cdots, L, \quad k, j=1, \cdots, J .
\end{gathered}
$$

\footnotetext{
${ }^{7}$ We put $J_{v}=0$, taking only left excitations in the pricipal chiral field language. We take both types of excitations into account in Appendix C. The full compact $S O(6)$ subsector is considered in Appendix E.
} 
where $M=\frac{g}{\sqrt{2}}$. These equations should be accompanied by a prescription that $\rho_{\theta}(z)$ has support $[-2,2]$ and has $1 / \sqrt{2 \pm z}$ behaviour near edges of the support. This prescription follows directly from eq. (10) in the $L \rightarrow \infty$ limit. For more details see [2].

The big parameter $g$ enters these equations thus inciting us to expand w.r.t. $1 / g$. But to reproduce the AFS equations we do not do it. We could even assume that $g$ is finite, thus imposing the rectangular shape of the potential with the finite length $2 \sqrt{2} g$. This is not possible with the massive relativistic dispersion eq. (13), where only the large $g$ limit leads to the rectangular shape of the potential, but it might be the adequate choice of dispersion in the full string theory, which should have the conformal symmetry in the conformal gauge. The similarity with the BDS equation where $g$ is small, and not big, supports this idea.

\subsection{Density}

Let us now calculate the density of distribution of $\theta$ 's. Taking log of the eq. (29) we obtain:

$$
\mathcal{G}_{\theta}(z)+2 \pi m=\sum_{j=1}^{K} i \log \frac{M z-u_{j}-i / 2}{M z-u_{j}+i / 2}
$$

Where

$$
G_{\theta}(z)=\frac{1}{M} \sum_{\alpha} \frac{1}{z-\theta_{\alpha} / M}=\int_{-2}^{2} \frac{d z^{\prime} \rho_{\theta}\left(z^{\prime}\right)}{z-z^{\prime}}
$$

and $\phi_{\theta}(z)$ is a real part of $G_{\theta}(z)$. We can find $G_{\theta}(z)$ as a function of $u_{j}$.

Performing the inverse Zhukovsky map eq.(22) we obtain the equation

$$
\mathcal{G}_{\theta}(z)+2 \pi m=i \sum_{j=1}^{K}\left(\log \frac{x-y_{j}^{+}}{x-y_{j}^{-}}+\log \frac{x-1 / y_{j}^{+}}{x-1 / y_{j}^{-}}\right)
$$

where $y_{j}^{ \pm}=X\left(\frac{u_{j} \pm i / 2}{M}\right), x=X(z) . X$ is defined in eq.(22).

Introducing

we obtain from eq. (33)

$$
H(z)=G_{\theta}(Z(x))
$$

$$
\frac{1}{2}[H(x)+H(1 / x)]=-2 \pi m+i \sum_{j=1}^{K}\left(\log \frac{x-y_{j}^{+}}{x-y_{j}^{-}}+\log \frac{x-1 / y_{j}^{+}}{x-1 / y_{j}^{-}}\right) .
$$

The solution of this equation, with the right asymptotics at infinity $H(1 / \epsilon)=G_{\theta}(1 / \epsilon) \simeq L / M \epsilon$, is as follows

$$
H(x)=\frac{\frac{L}{2 M}+2 \pi m}{x-1}+\frac{\frac{L}{2 M}-2 \pi m}{x+1}+i \sum_{j=1}^{K}\left[\frac{2 x}{x^{2}-1}\left(\frac{1}{y_{j}^{+}}-\frac{1}{y_{j}^{-}}\right)-\frac{2 x^{2} \log \frac{y_{j}^{+}}{y_{j}^{-}}}{x^{2}-1}+2 \log \frac{y_{j}^{+} x-1}{y_{j}^{-} x-1}\right]
$$

In 2] it was shown that in classical limit eqs. (16]17) can be expressed through poles of $H(x)$ in $x= \pm 1$. This result can be generalized for the case we consider there (see Appendix A). Extracting the residues of $H(x)$ at the poles $x= \pm 1$ we can see that

$$
\begin{aligned}
& \Delta=L+2 i M \sum_{j=1}^{K}\left(\frac{1}{y_{j}^{+}}-\frac{1}{y_{j}^{-}}\right) \\
& P=\left(m-\frac{i}{2 \pi} \sum_{j=1}^{K} \log \frac{y_{j}^{+}}{y_{j}^{-}}\right) \Delta=0
\end{aligned}
$$


eq.(37) is precisely the expression for the anomalous dimension eq. (26) and eq. (38) gives precisely the zero momentum condition for the AFS equation.

We can also compute the density of $\theta$ 's as the imaginary part of the resolvent $G_{\theta}(z)$

$$
\rho_{\theta}(Z(x))=\frac{\operatorname{Im} G_{\theta}(Z(x))}{\pi}=\frac{i}{2 \pi}[H(x)-H(1 / x)]
$$

\subsection{Derivation of AFS formula}

In this section we will exclude $\theta$ variables from (1112) using the $\theta$-density calculated above, and obtain the AFS equation eq. (20). We are trying here to go the same way as the authors of [17, where the similar variables were excluded in favor of the magnon variables in Lieb-Wu equations for the Hubbard model.

Let us now exclude $\theta$ 's from eq. (111), using the result eq.(36). Taking the log of eq.(111) we obtain

$$
\sum_{j \neq k} \log \frac{u_{k}-u_{j}+i}{u_{k}-u_{j}-i}+2 \pi i n_{k}=\sum_{\beta} \log \frac{u_{k}-\theta_{\beta}+i / 2}{u_{k}-\theta_{\beta}-i / 2} \equiv i p_{k}
$$

rewriting $p_{k}$ through density we have

$$
i p_{k}=M \int_{-2}^{2} \log \frac{z-w_{k}^{+}}{z-w_{k}^{-}} \rho_{\theta}(z) d z
$$

where $w_{k}^{ \pm}=\frac{u_{k} \pm i / 2}{M}$. The function $\rho_{\theta}(z)$ is given by eqs. (39)36). In Appendix A we perform the integration and obtain the following result

$$
\begin{aligned}
i p_{k} & =\sum_{j}\left[2 \log \frac{y_{k}^{-} y_{j}^{+}\left(y_{j}^{-} y_{k}^{+}-1\right)}{y_{k}^{+} y_{j}^{-}\left(y_{j}^{+} y_{k}^{-}-1\right)}-2 i\left(u_{j}-u_{k}\right) \log \frac{\left(y_{j}^{-} y_{k}^{-}-1\right)\left(y_{j}^{+} y_{k}^{+}-1\right)}{\left(y_{j}^{-} y_{k}^{+}-1\right)\left(y_{j}^{+} y_{k}^{-}-1\right)}\right] \\
& -2 M\left(\frac{1}{y_{k}^{+}}-\frac{1}{y_{k}^{-}}\right)\left[2 \pi m-i \sum_{j} \log \frac{y_{j}^{+}}{y_{j}^{-}}\right]+L \log \frac{y_{k}^{+}}{y_{k}^{-}}
\end{aligned}
$$

It leads to the following equations

$$
\left(\frac{y_{k}^{+}}{y_{k}^{-}}\right)^{L}=\prod_{j \neq k}^{K} \frac{y_{k}^{+}-y_{j}^{-}}{y_{k}^{-}-y_{j}^{+}}\left(\frac{1-1 /\left(y_{j}^{-} y_{k}^{+}\right)}{1-1 /\left(y_{j}^{+} y_{k}^{-}\right)}\right)^{-1}\left(\frac{\left(y_{j}^{-} y_{k}^{-}-1\right)}{\left(y_{j}^{-} y_{k}^{+}-1\right)} \frac{\left(y_{j}^{+} y_{k}^{+}-1\right)}{\left(y_{j}^{+} y_{k}^{-}-1\right)}\right)^{2 i\left(u_{j}-u_{k}\right)}
$$

which precisely coincide with the AFS [1] (20), including the expressions for energy and momentum eqs. (37/38).

\subsection{Periodicity in the world sheet momentum}

Denoting $\mathrm{p}_{\mathrm{j}}=-i \log \frac{y_{j}^{+}}{y_{j}^{-}}$, where $y^{ \pm}=X\left(\frac{u(\mathrm{p}) \pm i / 2}{M}\right)$, we rewrite eq. (21) in the way originally proposed in [1]

$$
\sigma^{2}\left(u_{j}, u_{k}\right)=\exp \left(2 i \sum_{r=2}^{\infty} M^{2 r}\left(q_{r}\left(\mathrm{p}_{k}\right) q_{r+1}\left(\mathrm{p}_{j}\right)-q_{r}\left(\mathrm{p}_{j}\right) q_{r+1}\left(\mathrm{p}_{k}\right)\right)\right)
$$

The functions $q_{r}(\mathrm{p})$ (charges) are given by

$$
q_{r}(\mathrm{p})=M^{2-2 r} \frac{2 \sin \left[\frac{1}{2}(r-1) \mathrm{p}\right]}{r-1}\left(\frac{\sqrt{1+16 M^{2} \sin ^{2}\left(\frac{1}{2} \mathrm{p}\right)}}{4 \sin \left(\frac{1}{2} \mathrm{p}\right)}\right)^{r-1}=i \frac{M^{-r+1}}{r-1}\left(y_{+}^{1-r}-y_{-}^{1-r}\right)
$$


We used here that from the definition of momentum $\mathrm{p}$ it follows

$$
u(\mathrm{p})=\frac{1}{2} \cot \frac{\mathrm{p}}{2} \sqrt{1+16 M^{2} \sin ^{2} \frac{\mathrm{p}}{2}}
$$

The energy and momentum eqs.(37/38) look now as follows

$$
\begin{aligned}
\Delta-L & =\sum_{j=1}^{K}\left(\sqrt{1+16 M^{2} \sin ^{2}\left(\mathrm{p}_{j} / 2\right)}-1\right) \\
P & =\left(m+\frac{\sum_{j} \mathrm{p}_{j}}{2 \pi}\right) \Delta=0,
\end{aligned}
$$

Thus by integrating out $\theta$ 's we obtain, instead of the relativistic dispersion relations (1617), the lattice dispersion relations in the effective magnon momentum defined through the l.h.s of eq. (43) as a free phase of the magnon: $e^{-i \mathrm{p}_{k}}=\left(\frac{y_{k}^{+}}{y_{k}^{-}}\right)$. All these formulas are periodic with respect to the magnon momentum $\mathrm{p}$, which was inspired in the AFS construction by the SYM spin chain.

On the SYM side, this dispersion relation was obtained in 36 in large $g$ limit and was than reproduced on the basis of integrability and supersymmetry in [16] [17. On the string side, the large $\lambda$ limit of this dispersion relation was reproduced in [24] considering the "giant magnon" configuration of the string.

\section{Discussion}

In this paper we derived the asymptotic string Bethe ansatz, AFS equations, directly from a more fundamental model of inhomogeneous dynamical spin chain (IDSC). The generalization to the full superstring theory is left to be guessed, and it will probably constrain further the possible properties of the IDSC model. The unknown dispersion relation for the "particles" constituting the chain, as well the scalar factor $S_{0}$ might become some functions of $\lambda$ and could have just a different behavior at strong and week coupling. Hopefully it will correspond to the known perturbative SYM and the quasiclassical string data. This is a possible way to reconcile the apparent differences of two theories, and in particular, to resolve the annoying 3-loop discrepancy. The methods of the direct calculation the bare string S-matrix of [32, as well as the results of [33] might help a lot.

Let us also note that the periodicity in momentum of elementary "magnon" excitations in the string theory discussed recently in 24] follow naturally from our construction since we reproduced the AFS equation. This periodicity, revealing the lattice structure of the IDSC model, stems in our approach from the specific distribution of $\theta$-variables: they are confined in a square box and the density has a characteristic inverse square root behavior at the end points.

For example, in the absence of magnons the density of $\theta$-variables is given by

$$
\rho_{0}(\theta) d \theta=\frac{L}{\pi} \frac{d \theta}{\sqrt{2 g^{2}-\theta^{2}}}, \quad \theta \in(-\sqrt{2} g, \sqrt{2}) .
$$

In terms of the variable $\theta=\sqrt{2} g \sin q$ this density simply becomes constant on a circle, like in the similar occasion in Hubbard model (see the Appendix of [17]):

$$
\rho_{q}(q) d q=\frac{L}{\pi} d q, \quad q \in(0,2 \pi) .
$$

The periodicity is a simple consequence of this behavior of the density.

It is interesting to note that taking in eq. (27)-(28)

$$
p\left(\theta_{\alpha}\right)=L\left(\arcsin \frac{\theta_{\alpha}}{\sqrt{2} g}-\phi\right)
$$


and

$$
S_{0}(\theta)=-1
$$

we obtain the Lieb-Wu equations for the Hubbard model with the energy of a state given by

$$
E=\frac{1}{g^{2}} \sum_{\alpha=1}^{L} \sqrt{2 g^{2}-\theta_{\alpha}^{2}}
$$

which reproduces, according to [28, the 3-loop (and may be all loop) anomalous dimensions $\Delta=E-L$. It is particularly clear in terms of the variable $q$.

The bound states, "strings", of a few magnons observed in 25] can be also naturally incorporated into the IDSC model $^{8}$.

An important check for the relevance of the IDSC model for the description of the GSMT superstring could come from the calculations of quantum $1 / \sqrt{\lambda}$ corrections. The discreteness of $\theta$ variables should give some specific contributions to the first correction to the energy [35, 34], together with the contributions from the discreteness of magnons calculated in [12, 13, 22, 21, 23.

An important unresolved question is the generalization of our construction to the full superstring theory. The "particles" out of which we make our inhomogeneous dynamical chain are yet to be identified in the full theory. An important guess might come from the system of asymptotic string AFS-type equations for the full superstring theory written in 14 .

\section{Acknowledgements}

We would like to thank I. Kostov, K, Sakai, D. Serban, A. Tseytlin, A. and Al. Zamolodchikovs, K. Zarembo and especially P. Vieira for discussions. The work of V.K. was partially supported by European Union under the RTN contracts MRTN-CT-2004-512194 and by INTAS-03-51-5460 grant. The work of N.G. was partially supported by French Government PhD fellowship and by RSGSS-1124.2003.2.

\section{Appendix A, Formula for energy}

In this appendix we derive formula for energy eq. (37) directly from eq. (17). In fact it is a trivial generalization of the result of [2, but we include it for completeness.

As it was shown in [2] density of $\theta$ 's behaves as $1 / \sqrt{2 \pm z}$ for $z \sim \mp 2$. We define $\kappa_{ \pm}$as follows

$$
\rho \simeq \frac{2 \kappa_{ \pm}}{\sqrt{2 \mp z}}, \quad z \rightarrow \pm 2
$$

We want to compute the sum

$$
E \equiv \frac{\mu}{2 \pi} \sum_{\alpha} \cosh \left(\pi \theta_{\alpha}\right),
$$

but we cannot simply replace this sum by an integral and use the asymptotic density for $\theta$ 's to compute the energy. This is because the main contribution for the energy comes from large $\theta$ 's, near the walls, where the expression for the asymptotic density is no longer accurate.

We notice that the energy is dominated by large $\theta$ 's where, with exponential precision, we can replace $\cosh \pi \theta_{\alpha}$ by $\pm \sinh \pi \theta_{\alpha}$ for positive (negative) $\theta_{\alpha}$. Then,

$$
E=\sum_{z_{\alpha}>0} \frac{\mu}{\pi} \sinh \left(\pi z_{\alpha} M\right)-\sum_{z_{\alpha}<0} \frac{\mu}{\pi} \sinh \left(\pi z_{\alpha} M\right),
$$

\footnotetext{
${ }^{8}$ We thank P.Vieira for pointing it out to us.
} 
Where $z_{\alpha}=\theta_{\alpha} / M$. Having a sum of $\sinh \pi \theta_{\alpha}$ we can substitute each of them by the corresponding Bethe equation (10) obtaining

$$
E \simeq \frac{i}{\pi} \sum_{z_{\beta}<0<z_{\alpha}} \log S_{0}^{2}\left(M\left[z_{\alpha}-z_{\beta}\right]\right)+\sum_{j, \alpha} \frac{i \operatorname{sign}\left(z_{\alpha}\right)}{\pi} \log \frac{z_{\alpha}-w_{j}^{-}}{z_{\alpha}-w_{j}^{+}}+\sum_{\alpha} m \operatorname{sign}\left(z_{\alpha}\right) .
$$

Where $w_{j}^{ \pm}=\frac{u_{j} \pm i / 2}{M}$. Now we can safely go to the continuous limit since in the first term the distances between $\xi$ 's are now mostly of order the 1 . This allows to rewrite the energy, with $1 / M$ precision, as follows

$$
\begin{aligned}
E & \simeq-\frac{M}{\pi} \int_{-2}^{a} d z \int_{a}^{2} d w \frac{\rho_{\theta}(z) \rho_{\theta}(w)}{z-w}+\frac{i M}{\pi} \sum_{j} \int \rho_{\theta}(z) \log \frac{z-w_{j}^{-}}{z-w_{j}^{+}} \operatorname{sign}(z-a) d z \\
& +m M \int \rho_{\theta}(w) \operatorname{sign}(w-a) d w
\end{aligned}
$$

where $a=0$. But now, due to eq. (29), one can see that we can take any $a \in(-2,2)$. Indeed, taking a derivative of r.h.s. of eq.(55) with respect to $a$, using eq.(29), we get zero. Hence we can even send $a$ close to the wall: $a=-2+\epsilon$, where $\epsilon$ is very small. Let us calculate the first term. The main contribution to the integral comes from $-2 \simeq z \sim w$ so that we can use the asymptotics (54) to get

$$
-\frac{M}{\pi} \int_{-2}^{-2+\epsilon} d z \int_{-2+\epsilon}^{2} d w \frac{\rho_{\theta}(z) \rho_{\theta}(w)}{z-w} \simeq-\int_{-2}^{-2+\epsilon} d z \int_{-2+\epsilon}^{2} d w \frac{4 M \kappa_{-}^{2}}{\pi(z-w) \sqrt{2+z} \sqrt{2+w}} \simeq 2 \pi M \kappa_{-}^{2}
$$

The remaining 3 terms are very simple: since $a \simeq-2$ we can simply drop the sign-functions inside the integrals and obtain exactly the expression of the momentum in the continuous limit. We arrive therefore at

$$
E \simeq 2 M \kappa_{-}^{2} \pi+\left(m L-\sum_{p} n_{p} J_{p}\right)
$$

where $K_{p}$ is a number of $u$ 's with mode number $n_{p}$. If we compute the $a$-independent integral (55) near the other wall, i.e. for $a=2-\epsilon$, we find

$$
E \simeq 2 M \kappa_{+}^{2} \pi-\left(m L-\sum_{p} n_{p} J_{p}\right) .
$$

Therefore, equating the results one obtains the desired expressions for the energy and momentum

$$
E \pm P=2 \pi M \kappa_{ \pm}^{2}
$$

through the data $\kappa_{ \pm}$at the singularities of the curve at $z= \pm 2$. From eq. (557) we see that $\kappa_{+}=\kappa_{-}=\kappa$ to ensure $P=0$. We can also write

$$
\Delta=\lambda^{1 / 4} \sqrt{2 E}=4 \pi M \kappa
$$

and we immediately get eqs.(37/38) from eq.(36).

\section{Appendix B, Derivation of AFS formula for asymptotic string BAE's}

In this appendix we evaluate integral (41) and obtain AFS BAE.

We can simplify expression for $H(x)$ (36) assuming that in eq.(38) $P=0$

$$
H(x)=-4 \pi m+\frac{\Delta}{M} \frac{x}{x^{2}-1}+2 i \sum_{j} \log \frac{y_{j}^{+} x-1}{y_{j}^{-} x-1}
$$


we rewrite (41) in $x$ variable

$$
i p_{k}=-\frac{M}{2} \oint \frac{i}{2 \pi}(H(x)-H(1 / x))\left(\log \frac{x-y_{k}^{+}}{x-y_{k}^{-}}+\log \frac{x-1 / y_{k}^{+}}{x-1 / y_{k}^{-}}\right)\left(1-\frac{1}{x^{2}}\right) d x
$$

where contour goes in counterclockwise direction around unit circle, $y_{k}^{ \pm}=X\left(w_{k}^{ \pm}\right)$. Note that terms with $H(1 / x)$ are equal to that with $H(x)$ after change of the variable $x \rightarrow 1 / x$. So that

$$
i p_{k}=M \oint H(x)\left(\log \frac{x-y_{k}^{+}}{x-y_{k}^{-}}+\log \frac{x-1 / y_{k}^{+}}{x-1 / y_{k}^{-}}\right)\left(\frac{x^{2}-1}{x^{2}}\right) \frac{d x}{2 \pi i}
$$

Various terms are

$$
\begin{aligned}
I_{1} & \equiv \oint\left(-4 \pi m+\frac{\Delta}{M} \frac{x}{x^{2}-1}\right) \log \frac{x-y_{k}^{+}}{x-y_{k}^{-}}\left(\frac{x^{2}-1}{x^{2}}\right) \frac{d x}{2 \pi i} \\
I_{2} & \equiv \oint\left(-4 \pi m+\frac{\Delta}{M} \frac{x}{x^{2}-1}\right) \log \frac{x-1 / y_{k}^{+}}{x-1 / y_{k}^{-}}\left(\frac{x^{2}-1}{x^{2}}\right) \frac{d x}{2 \pi i} \\
I_{3} & \equiv 2 i \oint \log \frac{y_{j}^{+} x-1}{y_{j}^{-} x-1} \log \frac{x-y_{k}^{+}}{x-y_{k}^{-}}\left(\frac{x^{2}-1}{x^{2}}\right) \frac{d x}{2 \pi i} \\
I_{4} & \equiv 2 i \oint \log \frac{y_{j}^{+} x-1}{y_{j}^{-} x-1} \log \frac{x-1 / y_{k}^{+}}{x-1 / y_{k}^{-}}\left(\frac{x^{2}-1}{x^{2}}\right) \frac{d x}{2 \pi i}
\end{aligned}
$$

Integral $I_{1}$ can be calculated by residue in $x=0$, since $\left|y_{k}^{ \pm}\right|>1$.

$$
I_{1}=\frac{\Delta}{M} \log \frac{y_{k}^{+}}{y_{k}^{-}}-4 \pi m\left(\frac{1}{y_{k}^{+}}-\frac{1}{y_{k}^{-}}\right)
$$

Similar $I_{2}$ and $I_{4}$ are given by residue at infinity.

$$
\begin{aligned}
& I_{2}=4 \pi m\left(\frac{1}{y_{k}^{+}}-\frac{1}{y_{k}^{-}}\right) \\
& I_{4}=-2 i\left(\frac{1}{y_{k}^{+}}-\frac{1}{y_{k}^{-}}\right) \log \frac{y_{j}^{+}}{y_{j}^{-}}
\end{aligned}
$$

Calculation of $I_{3}$ is slightly more difficult. One can differentiate it with respect to $y_{j}^{+}$to kill one of the logarithms and then calculate it by poles at $x=0$

$$
\partial_{y_{j}^{+}} I_{3}=2 i \log \frac{y_{k}^{+}}{y_{k}^{-}}+2 i\left(\frac{1}{y_{j}^{+^{2}}}-1\right) \log \frac{y_{k}^{+} y_{j}^{+}-1}{y_{k}^{-} y_{j}^{+}-1}, \quad I_{3}=\int_{y_{j}^{-}}^{y_{j}^{+}} \partial_{y_{j}^{+}} I_{3} d y_{j}^{+}
$$

thus

$$
I_{3}=2 i \frac{u_{j}-u_{k}}{M} \log \frac{\left(y_{j}^{+} y_{k}^{-}-1\right)\left(y_{j}^{-} y_{k}^{+}-1\right)}{\left(y_{j}^{+} y_{k}^{+}-1\right)\left(y_{j}^{-} y_{k}^{-}-1\right)}+\frac{2}{M} \log \frac{y_{j}^{-} y_{k}^{+}-1}{y_{j}^{+} y_{k}^{-}-1}+2 i\left(\left(y_{j}^{+}-y_{j}^{-}\right) \log \frac{y_{k}^{+}}{y_{k}^{-}}-\left(y_{k}^{+}-y_{k}^{-}\right) \log \frac{y_{j}^{+}}{y_{j}^{-}}\right)
$$

Finally

$$
i p_{k}=M \sum_{a=1}^{4} I_{a}=L \log \frac{y_{k}^{+}}{y_{k}^{-}}+\sum_{j}\left(2 \log \frac{1-1 / y_{j}^{-} y_{k}^{+}}{1-1 / y_{j}^{+} y_{k}^{-}}+2 i\left(u_{j}-u_{k}\right) \log \frac{\left(y_{j}^{+} y_{k}^{-}-1\right)\left(y_{j}^{-} y_{k}^{+}-1\right)}{\left(y_{j}^{+} y_{k}^{+}-1\right)\left(y_{j}^{-} y_{k}^{-}-1\right)}\right)
$$

thus we prove eq.(42) assuming $P=0$. This immediately leads to AFS BAE eq.(43). 


\section{Appendix C, Full $S^{3} \times R$ sector}

We can easily integrate out $\theta$ 's variables in the case of both nontrivial chiralities $J_{u}, J_{v} \neq 0$. Resolvent of the $\theta$ 's becomes

$$
H(x)=\frac{\frac{L}{2 M}+2 \pi m}{x-1}+\frac{\frac{L}{2 M}-2 \pi m}{x+1}+i \sum_{j=1}^{K_{u}+K_{v}}\left[\frac{2 x}{x^{2}-1}\left(\frac{1}{y_{j}^{+}}-\frac{1}{y_{j}^{-}}\right)-\frac{2 x^{2} \log \frac{y_{j}^{+}}{y_{j}^{-}}}{x^{2}-1}+2 \log \frac{y_{j}^{+} x-1}{y_{j}^{-} x-1}\right]
$$

where we denoted

$$
\begin{aligned}
y_{j}^{ \pm}=X\left(\frac{u_{j} \pm i / 2}{M}\right), \quad j=1, \ldots, K_{u} \\
y_{l+K_{u}}^{ \pm} \equiv \tilde{y}_{l}^{ \pm}=X\left(\frac{v_{l} \pm i / 2}{M}\right), \quad l=1, \ldots, K_{v}
\end{aligned}
$$

Formulas for energy and momentum thus have the same form eqs.(3738). $u$ 's and v's don't entangle. For magnon momentum eq. (41) we obviously have

$$
i p_{k}=\sum_{j=1}^{K_{u}+K_{v}}\left[2 \log \frac{y_{k}^{-} y_{j}^{+}\left(y_{j}^{-} y_{k}^{+}-1\right)}{y_{k}^{+} y_{j}^{-}\left(y_{j}^{+} y_{k}^{-}-1\right)}-2 i\left(u_{j}-u_{k}\right) \log \frac{\left(y_{j}^{-} y_{k}^{-}-1\right)\left(y_{j}^{+} y_{k}^{+}-1\right)}{\left(y_{j}^{-} y_{k}^{+}-1\right)\left(y_{j}^{+} y_{k}^{-}-1\right)}\right]+L \log \frac{y_{k}^{+}}{y_{k}^{-}}
$$

and thus BAE takes the form

$$
\begin{aligned}
\left(\frac{y_{k}^{+}}{y_{k}^{-}}\right)^{L} & =\prod_{j=1}^{K_{u}} \frac{y_{k}^{+}-y_{j}^{-}}{y_{k}^{-}-y_{j}^{+}}\left(\frac{1-1 /\left(y_{j}^{-} y_{k}^{+}\right)}{1-1 /\left(y_{j}^{+} y_{k}^{-}\right)}\right)^{-1}\left(\frac{\left(y_{j}^{-} y_{k}^{-}-1\right)}{\left(y_{j}^{-} y_{k}^{+}-1\right)} \frac{\left(y_{j}^{+} y_{k}^{+}-1\right)}{\left(y_{j}^{+} y_{k}^{-}-1\right)}\right)^{2 i\left(u_{j}-u_{k}\right)} \times \\
& \times \prod_{l=1}^{K_{v}}\left(\frac{1-1 /\left(\tilde{y}_{l}^{-} y_{k}^{+}\right)}{1-1 /\left(\tilde{y}_{l}^{+} y_{k}^{-}\right)}\right)^{-2}\left(\frac{\left(\tilde{y}_{l}^{-} y_{k}^{-}-1\right)}{\left(\tilde{y}_{l}^{-} y_{k}^{+}-1\right)} \frac{\left(\tilde{y}_{l}^{+} y_{k}^{+}-1\right)}{\left(\tilde{y}_{l}^{+} y_{k}^{-}-1\right)}\right)^{2 i\left(v_{l}-u_{k}\right)}
\end{aligned}
$$

and symmetrical equation for $v$ 's.

\section{Appendix D, $S^{5} \times R$ sector}

We can also generalize then calculation of Appendix $\mathrm{C}$ on $S O(6)$ case. Full BAE for this case are

$$
\begin{aligned}
e^{-i \mu \sinh \frac{\pi \theta_{\alpha}}{2}} & =\prod_{\beta \neq \alpha}^{L} S_{0}\left(\theta_{\alpha}-\theta_{\beta}\right) \prod_{j=1}^{K_{2}} \frac{\theta_{\alpha}-u_{j}^{(2)}+i / 2}{\theta_{\alpha}-u_{j}^{(2)}-i / 2} \\
1 & =\prod_{j \neq i}^{K_{1}} \frac{u_{i}^{(1)}-u_{j}^{(1)}+i}{u_{i}^{(1)}-u_{j}^{(1)}-i} \prod_{j=1}^{K_{2}} \frac{u_{i}^{(1)}-u_{j}^{(2)}-i / 2}{u_{i}^{(1)}-u_{j}^{(2)}+i / 2} \\
\prod_{\alpha=1}^{L} \frac{u_{i}^{(2)}-\theta_{\alpha}+i / 2}{u_{i}^{(2)}-\theta_{\alpha}-i / 2}= & \prod_{j \neq i}^{K_{2}} \frac{u_{i}^{(2)}-u_{j}^{(2)}+i}{u_{i}^{(2)}-u_{j}^{(2)}-i} \prod_{j=1}^{K_{3}} \frac{u_{i}^{(2)}-u_{j}^{(3)}-i / 2}{u_{i}^{(2)}-u_{j}^{(3)}+i / 2} \prod_{j=1}^{K_{1}} \frac{u_{i}^{(2)}-u_{j}^{(1)}-i / 2}{u_{i}^{(2)}-u_{j}^{(1)}+i / 2} \\
1= & \prod_{j \neq i}^{K} \frac{u_{i}^{(3)}-u_{j}^{(3)}+i}{u_{i}^{(3)}-u_{j}^{(3)}-i} \prod_{j=1}^{K_{2}} \frac{u_{i}^{(3)}-u_{j}^{(2)}-i / 2}{u_{i}^{(3)}-u_{j}^{(2)}+i / 2}
\end{aligned}
$$

where

$$
S_{0}(\theta)=-\frac{\Gamma\left(\frac{1}{4}-i \frac{\theta}{4}\right) \Gamma\left(\frac{1}{2}-i \frac{\theta}{4}\right) \Gamma\left(\frac{3}{4}+i \frac{\theta}{4}\right) \Gamma\left(1+i \frac{\theta}{4}\right)}{\Gamma\left(\frac{1}{4}+i \frac{\theta}{4}\right) \Gamma\left(\frac{1}{2}+i \frac{\theta}{4}\right) \Gamma\left(\frac{3}{4}-i \frac{\theta}{4}\right) \Gamma\left(1-i \frac{\theta}{4}\right)} .
$$


and for large $\lambda$ one should take $\mu=\epsilon^{-\frac{\sqrt{\lambda}}{4}}$. From the first line in eq. w6 wee that denoting

$$
i p_{k}=M \int_{-2}^{2} \log \frac{z M-u_{k}^{(2)}-i / 2}{z M-u_{k}^{(2)}+i / 2} \rho_{\theta}(z) d z
$$

we again have eq. (42) with $y_{j}^{ \pm}=X\left(\frac{u_{j}^{(2)} \pm i / 2}{M}\right)$ and thus we have the following set of effective BAE equations for large $L \sim \sqrt{\lambda}$

$$
\begin{aligned}
1 & =\prod_{j \neq i}^{K_{1}} \frac{u_{i}^{(1)}-u_{j}^{(1)}+i}{u_{i}^{(1)}-u_{j}^{(1)}-i} \prod_{j=1}^{K_{2}} \frac{u_{i}^{(1)}-u_{j}^{(2)}-i / 2}{u_{i}^{(1)}-u_{j}^{(2)}+i / 2} \\
\left(\frac{y_{i}^{+}}{y_{i}^{-}}\right)^{L} & =\prod_{j \neq i}^{K_{2}}\left(\frac{u_{i}^{(2)}-u_{j}^{(2)}+i}{u_{i}^{(2)}-u_{j}^{(2)}-i} \sigma^{2}\left(u_{j}, u_{i}\right)\right) \prod_{j=1}^{K_{3}} \frac{u_{i}^{(2)}-u_{j}^{(3)}-i / 2}{u_{i}^{(2)}-u_{j}^{(3)}+i / 2} \prod_{j=1}^{K_{1}} \frac{u_{i}^{(2)}-u_{j}^{(1)}-i / 2}{u_{i}^{(2)}-u_{j}^{(1)}+i / 2} \\
1 & =\prod_{j \neq i}^{K_{3}} \frac{u_{i}^{(3)}-u_{j}^{(3)}+i}{u_{i}^{(3)}-u_{j}^{(3)}-i} \prod_{j=1}^{K_{2}} \frac{u_{i}^{(3)}-u_{j}^{(2)}-i / 2}{u_{i}^{(3)}-u_{j}^{(2)}+i / 2}
\end{aligned}
$$

\section{Appendix E, Classical limit}

Having at hands explicit formula for resolvent eq. (36) we can shortly show how the classical equations of [5] appears from (10 12). Classical limit corresponds to large number of excitations $J_{u}, J_{v} \sim L \sim M$ and finite mode numbers $n_{i}^{u}, n_{i}^{v} \sim 1$. In this limit Bethe roots scale as $M$. We denote

$$
w_{i}=\frac{u_{i}}{M}, \quad \tilde{w}_{i}=\frac{v_{i}}{M}, \quad y_{i}=X\left(\frac{u_{i}}{M}\right), \quad \tilde{y}_{i}=X\left(\frac{v_{i}}{M}\right)
$$

The resolvent eq. (71) in this limit becomes

$$
H(x)=\frac{\frac{L}{M} x+4 \pi m}{x^{2}-1}-\frac{2}{x^{2}-1} \frac{1}{M} \sum_{j=1}^{K_{u}+K_{v}} \frac{1}{1 / x-y_{j}}
$$

Expanding eq. (11) we have

$$
\pi n_{k}=-\frac{1}{2} H\left(y_{k}\right)-\frac{1}{y_{k}^{2}-1} \frac{1}{M} \sum_{j \neq k}^{K_{u}} \frac{1}{1 / y_{k}-y_{j}}+\frac{y_{k}^{2}}{y_{k}^{2}-1} \frac{1}{M} \sum_{j \neq k}^{K_{u}} \frac{1}{y_{k}-y_{j}}
$$

Using eq. (81) and dropping $\mathcal{O}(1 / M)$ terms we have

$$
\pi n_{k}=\frac{\frac{L}{2 M} y_{k}+2 \pi m}{1-y_{k}^{2}}+\frac{1}{y_{k}^{2}-1} \frac{1}{M} \sum_{j=1}^{K_{v}} \frac{1}{1 / y_{k}-\tilde{y}_{j}}+\frac{y_{k}^{2}}{y_{k}^{2}-1} \frac{1}{M} \sum_{j \neq k}^{K_{u}} \frac{1}{y_{k}-y_{j}}
$$

Defining [2]

$$
p(x)=\frac{\frac{L}{2 M} x+2 \pi m}{1-x^{2}}+\frac{1}{x^{2}-1} \frac{1}{M} \sum_{j=1}^{K_{v}} \frac{1}{1 / x-\tilde{y}_{j}}+\frac{x^{2}}{x^{2}-1} \frac{1}{M} \sum_{j=1}^{K_{u}} \frac{1}{x-y_{j}}
$$

we can see that $p(x)$ satisfy classical equations of KMMZ [5], namely

$$
\begin{aligned}
& p(1+\epsilon) \simeq p(-1+\epsilon) \simeq-\frac{\Delta}{4 M \epsilon} \\
& p(\epsilon)=2 \pi m+\frac{\epsilon}{2 M}\left(L-2 J_{v}\right) \\
& p(1 / \epsilon)=-\frac{\epsilon}{2 M}\left(L-2 J_{u}\right) \\
& \not p(x)=\pi n_{j}, \quad x \in \mathcal{C}
\end{aligned}
$$


where $M=\sqrt{\lambda} / 4 \pi, \Delta=\sqrt{\lambda} \kappa$.

\section{References}

[1] G. Arutyunov, S. Frolov and M. Staudacher, "Bethe ansatz for quantum strings," JHEP 0410, 016 (2004) arXiv:hep-th/0406256.

[2] N. Gromov, V. Kazakov, K. Sakai and P. Vieira, "Strings as multi-particle states of quantum sigmamodels," arXiv:hep-th/0603043.

[3] A. B. Zamolodchikov and A. B. Zamolodchikov, "Relativistic Factorized S Matrix In Two-Dimensions Having O(N) Isotopic Symmetry,” Nucl. Phys. B 133 (1978) 525 [JETP Lett. 26 (1977) 457].

[4] N. Mann and J. Polchinski, "Bethe ansatz for a quantum supercoset sigma model," Phys. Rev. D 72 (2005) 086002 arXiv:hep-th/0508232.

[5] V. A. Kazakov, A. Marshakov, J. A. Minahan and K. Zarembo, "Classical / quantum integrability in $A d S / C F T "$, JHEP 0405, 024 (2004) arXiv:hep-th/0402207.

[6] N. Beisert, V. A. Kazakov and K. Sakai, "Algebraic curve for the SO(6) sector of AdS/CFT," arXiv:hep-th/0410253

[7] D. Berenstein, J. M. Maldacena and H. Nastase, "Strings in flat space and pp waves from $N=4$ super Yang Mills," JHEP 0204 (2002) 013 arXiv:hep-th/0202021.

[8] C. G. . Callan, H. K. Lee, T. McLoughlin, J. H. Schwarz, I. J. Swanson and X. Wu, "Quantizing string theory in AdS(5) x $S^{* *} 5$ : Beyond the pp-wave," Nucl. Phys. B 673, 3 (2003) arXiv:hep-th/0307032.

[9] S. Frolov and A. A. Tseytlin, "Semiclassical quantization of rotating superstring in AdS(5) x S(5)," JHEP 0206 (2002) 007 arXiv:hep-th/0204226.

[10] S. Frolov and A. A. Tseytlin, "Multi-spin string solutions in AdS(5) x $S^{* * 5}$," Nucl. Phys. B 668 (2003) 77 arXiv:hep-th/0304255.

[11] I. Y. Park, A. Tirziu and A. A. Tseytlin, "Spinning strings in AdS(5) $x S^{* * 5}$ : One-loop correction to energy in SL(2) sector," JHEP 0503 (2005) 013 arXiv:hep-th/0501203.

[12] S. Schafer-Nameki, M. Zamaklar and K. Zarembo, "Quantum corrections to spinning strings in AdS(5) $x S^{* * 5}$ and Bethe ansatz: A comparative study," JHEP 0509 (2005) 051 arXiv:hep-th/0507189.

[13] N. Beisert and A. A. Tseytlin, "On quantum corrections to spinning strings and Bethe equations," Phys. Lett. B 629, 102 (2005) arXiv:hep-th/0509084.

[14] N. Beisert and M. Staudacher, "Long-range PSU(2,2|4) Bethe ansaetze for gauge theory and strings," Nucl. Phys. B 727 (2005) 1 arXiv:hep-th/0504190.

[15] M. Staudacher, "The factorized S-matrix of CFT/AdS," JHEP 0505 (2005) 054 arXiv:hep-th/0412188.

[16] N. Beisert, V. Dippel and M. Staudacher, "A novel long range spin chain and planar $N=4$ super Yang-Mills," JHEP 0407 (2004) 075 arXiv:hep-th/0405001.

[17] A. Rej, D. Serban and M. Staudacher, "Planar $N=4$ gauge theory and the Hubbard model," arXiv:hep-th/0512077

[18] N. Beisert, "An SU(1|1)-invariant S-matrix with dynamic representations," arXiv:hep-th/0511013

[19] N. Beisert, "The su(2|2) dynamic S-matrix," arXiv:hep-th/0511082

[20] R. A. Janik, "The AdS(5) x $S^{* * 5}$ superstring worldsheet $S$-matrix and crossing symmetry," Phys. Rev. D 73 (2006) 086006 arXiv:hep-th/0603038. 
[21] R. Hernandez and E. Lopez, "Quantum corrections to the string Bethe ansatz," arXiv:hep-th/0603204

[22] G. Arutyunov and S. Frolov, "On AdS(5) x $S^{* * 5}$ string S-matrix," arXiv:hep-th/0604043.

[23] L. Freyhult and C. Kristjansen, "A universality test of the quantum string Bethe ansatz," arXiv:hep-th/0604069

[24] D. M. Hofman and J. Maldacena, "Giant magnons," arXiv:hep-th/0604135

[25] N. Dorey, "Magnon bound states and the AdS/CFT correspondence," arXiv:hep-th/0604175

[26] M. B. Green and J. H. Schwarz, "Supersymmetrical String Theories," Phys. Lett. B 109 (1982) 444.

[27] R. R. Metsaev and A. A. Tseytlin, "Type IIB superstring action in AdS(5) x S(5) background," Nucl. Phys. B 533 (1998) 109 arXiv:hep-th/9805028.

[28] D. Serban and M. Staudacher, "Planar $N=4$ gauge theory and the Inozemtsev long range spin chain," JHEP 0406 (2004) 001 arXiv:hep-th/0401057.

[29] N. Mann and J. Polchinski, "Finite density states in integrable conformal field theories," arXiv:hep-th/0408162

[30] N. Beisert, "Spin chain for quantum strings," Fortsch. Phys. 53, 852 (2005) arXiv:hep-th/0409054.

[31] N. Beisert, "The su(2|3) dynamic spin chain," Nucl. Phys. B 682, 487 (2004) arXiv:hep-th/0310252.

[32] T. Klose and K. Zarembo, "Bethe ansatz in stringy sigma models," arXiv:hep-th/0603039

[33] R. Roiban, A. Tirziu and A. A. Tseytlin, "Asymptotic Bethe Ansatz S-matrix and Landau-Lifshitz type effective 2-d actions," arXiv:hep-th/0604199.

[34] S. Schafer-Nameki and M. Zamaklar, "Stringy sums and corrections to the quantum string Bethe ansatz," JHEP 0510 (2005) 044 arXiv:hep-th/0509096.

[35] S. Schafer-Nameki, "Exact expressions for quantum corrections to spinning strings," arXiv:hep-th/0602214

[36] D. Berenstein, D. H. Correa and S. E. Vazquez, JHEP 0602, 048 (2006) arXiv:hep-th/0509015. 